\title{
Large anterior vaginal wall cyst in pregnancy
}

${ }^{1}$ Raguraman S, ${ }^{2}$ Dilinika MMS, ${ }^{2}$ Kanagallingam $A$

${ }^{1}$ DGH Mullaitivu and Faculty of Medicine, Jaffna, ${ }^{2}$ DGH Mullaitivu

\begin{abstract}
Vaginal wall cysts are uncommon and are broadly classified according to the lining epithelium of the cyst into epithelial inclusion cysts, embryonic (Müllerian and Gartner's) cysts and urothelial cysts. Müllerian cysts are the commonest congenital cysts of the vagina. We present a case report of a 36 yearold pregnant woman presented the first time in her life with anterior vaginal wall cyst on her second pregnancy. A systematic clinical approach such as history, examination and investigations (imaging studies) was an aid to plan out the management.
\end{abstract}

\section{Keywords}

Vaginal Cyst, Pregnancy, Mullerian cyst, Cyst aspiration, cyst excision.

\section{Introduction}

Cystic lesion of the vagina could occur anywhere in the vaginal wall, usually laterally and rarely extended to the fornix. Vaginal cysts have various types according to the histology such as Mullerian cysts, epidermal inclusion cysts, Gartner's duct cysts, Bartholin's gland cysts and endometriotic cysts (1). It is usually reported to present in the third and fourth decades of life. Meanwhile, a vaginal cyst could clinically present in several ways for instance; asymptomatic in the majority, vaginal protruding lump, discomfort during sex, tampon insertion \& walking and features of infections with discharge. Rarely, the vaginal cyst might initially present during pregnancy as a protruding lump. Only a few cases of vaginal cysts have been reported which have encountered in pregnancy or during labour (2)
We present a rare case of a large anterior vaginal wall cyst presented initially in woman during her second pregnancy.

\section{Case report}

A 36 year-old pregnant woman (gravida 2, para 1) presented to the antenatal clinic with a lump at vulva at 20 weeks of period of gestation (POG). It had developed for the first time in her life over four days with discomfort, but she did not have pain, discharge, bleeding, urinary symptoms nor bowel symptoms. Her first pregnancy was uneventful and had a normal vaginal delivery. Antenatal period of her second pregnancy was uneventful with exception of iron deficiency anaemia. The examination showed a lump (size- $4 \mathrm{~cm} \times 4 \mathrm{~cm}$ ) bulging from the anterior vaginal wall and was $2 \mathrm{~cm}$ away from the external urethral meatus. Ultrasound scan showed an anterior vaginal wall cyst, $6 \mathrm{~mm}$ away and lateral to the urethra.

At 32 weeks of the POG, she presented with a protruding lump with discomfort especially on straining and walking. The vaginal cyst size had increased to $6 \mathrm{~cm} \times 7 \mathrm{~cm}$. Due to discomfort and increasing size, vaginal wall cyst was aspirated and mucinous fluid was sent for cytological analysis. She was discharged following the cyst analysis which was normal. (Figure 1)

She presented again with large cyst with discomfort at 37 weeks without infective features. After clear explanation and counselling of both partners, to avoid the risk of pressure-rupture of the cyst or obliteration of the birth canal during labour elective Cesarean section and vaginal cyst excision was performed at term and a healthy baby was delivered. Histology report revealed the cyst as Mullerian cyst. (Figure 2) 


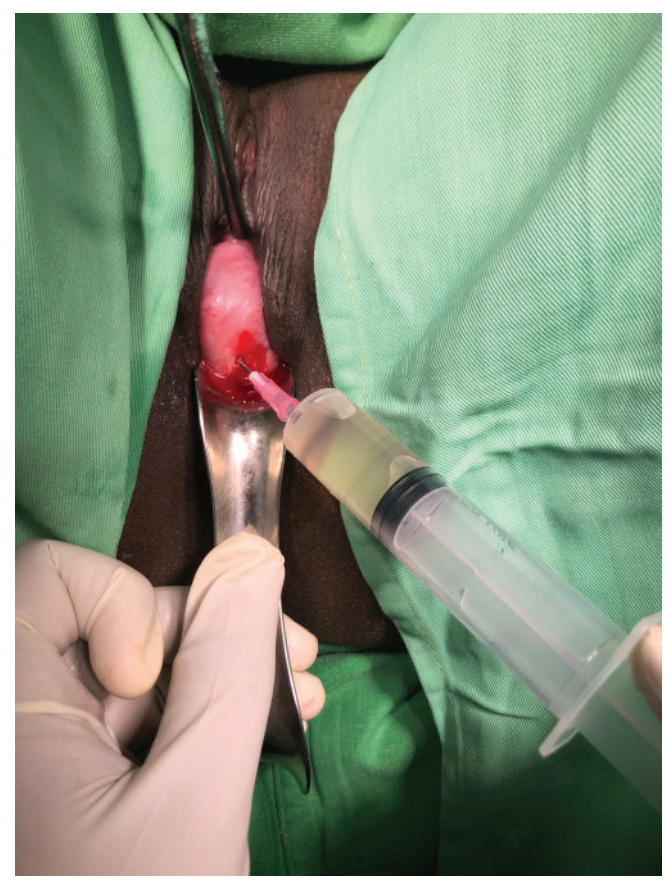

Figure 1

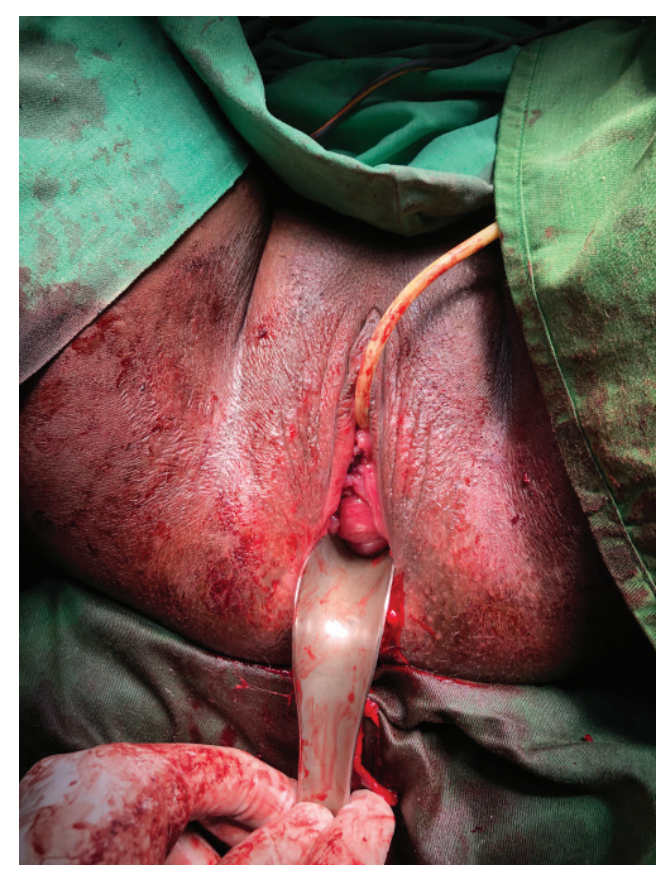

Figure 2

Figure 1-Aspiration of the cyst at 32 weeks of POG

Figure 2- After complete excision of the cyst following Caesarean section

\section{Discussion}

Prevalence of vaginal cyst, especially in pregnancy has been reported differently in several case reports. However, our literature survey suggested the prevalence of vaginal cysts has been estimated to be 1 in 200, but this might an underestimate as most vaginal cysts are not reported (2). There are several classifications of vaginal cyst according to their location, histology and embryonic origin. It has been classified according to the histological lining of the cyst as epidermal inclusion cysts, embryonic (Müllerian or Gartner's cysts) and urothelial cysts (3).

Müllerian cysts are the commonest congenital cysts of the vagina and present with different sizes. They are commonly located at the anterolateral vaginal wall, although a few multifocal Müllerian cysts have been also reported $(4,5)$. During the eighth week of embryologic development, the paired Müllerian (paramesonephric) ducts fuse distally and develop into the uterus, cervix and upper vagina, which are lined by a pseudostratified columnar (glandular) epithelium. Wolffian (mesonephric) ducts normally regress in the female. Müllerian and Wolffian derivatives can be found at almost any location within the vaginal walls.

Vaginal cysts are managed in several ways such as incision and drainage (I\&D), marsupialization and complete cyst excision depended on the clinical features and imaging study's findings. Vaginal cyst complicating pregnancies are usually managed conservatively during the antenatal period and complete surgical excision of the cyst following the delivery. However, symptomatic vaginal cysts are managed with cyst content aspiration during the antenatal period to relive the pressure effect by the vaginal cyst. Mode of delivery usually decided with obstetric indications but our literature search had shown several cases were managed by elective caesarian section due to cyst rupture and birth canal obstruction during $2^{\text {nd }}$ stage of labour (6).

In this case, she presented for the first time at her second pregnancy. Therefore, clinical assessment and imaging studies play a pivotal role in the management. As the aspiration fluid was negative for malignant cells MRI was not performed to 
exclude the malignant potential of the cyst. In literature, most of the vaginal cysts, especially late-onset were managed with the aid of MRI to exclude malignancies and extent of the cyst into the perineum (7). We delivered the healthy baby by elective caesarian section and following the delivery cyst was excised completely with unremarkable recovery.

\section{Conclusion}

Vaginal cyst complicating in pregnancy is a rare condition. Clinicians need a proper clinical assessment of the cyst and imaging studies such as USS and MRI to plan out the management specifically to exclude the very rare possibilities of malignant changes. Surgical intervention of cyst wall is possible with unremarkable recovery soon after the delivery. However, more case series need to be analysed to determine the definitive treatment options during the antenatal period.

\section{References}

1. Pradhan S, Tobon H. Vaginal cysts: a clinicopathological study of 41 cases. Int J Gynecol Pathol 1986; 5: 35-46.
2. Junaid TA, Thomas SM. Cysts of the vulva and vagina: a comparative study. Int J Gynaecol Obstet 1981; 19: 239-43

3. Kondi-Pafiti A, Grapsa D, Papakonstantinou K, KairiVassilatou E, Xasiakos D. Vaginal cysts: a common pathologic entity revisited. Clin Exp Obstet Gynecol 2008; 35: 41-44.

4. Wai CY, Corton MM, Miller M, et al. Multiple vaginal wall cysts: diagnosis and surgical management. Obstet Gynecol 2004; 103: 1099102.

5. Hwang JH, Oh MJ, Lee NW. Multiple Mullerian cysts: a case report and review of literature. Arch Gynecol Obstet 2009; 280: 137-9.

6. Lallar M, Nandal R, Sharma D, Shastri S: Large posterior vaginal cyst in pregnancy. BMJ Case Repots 2015 Jan; 20: 2015: 1-3. Available from http://dx.doi.org/10.1136/bcr-2014-208874

7. Y. GOLDBERG, O. LAVIE, R. MANDEL and R. AUSLENDER: Imaging of an atypical large perineal cyst diagnosed during pregnancy. Ultrasound Obstet Gynecol 2012; 40: 721-723. Available from https://doi.org/10.1002/uog.11130 